九州大学学術情報リポジトリ

Kyushu University Institutional Repository

\title{
Development of the Multilingual Collaboration System for Farmers of Several Countries (2): Multilingual Full Text Search System
}

Lee, Kang Oh

Laboratory of Agricultural Ecology, Division of Agricultural Ecology, Department of Plant Resource, Graduate School of Bioresoruce and Bioenvironmental Science, Kyushu University

Nakaji, Ke i

Laboratory of Agricultural Ecology, Division of Agricultural Ecology, Department of Plant Resource, Faculty of Agriculture, Kyushu University

Nada, Yoichi

Laboratory of Agricultural Ecology, Division of Agricultural Ecology, Department of Plant Resource, Graduate School of Bioresoruce and Bioenvironmental Science, Kyushu University

https://doi.org/10.5109/4605

出版情報: 九州大学大学院農学研究院紀要. 49 (2)，pp.441-448，2004-10-01. Faculty of Agriculture, Kyushu University

バージョン：

権利関係 : 
J. Fac. Agr., Kyushu Univ., 49 (2), 441-448 (2004)

\title{
Development of the Multilingual Collaboration System for Farmers of Several Countries (Part 2) - Multilingual Full Text Search System -
}

\author{
Kang Oh LEE ${ }^{1}$, Kei NAKAJI* and Yoichi NADA \\ Laboratory of Agricultural Ecology, Division of Agricultural Ecology, \\ Department of Plant Resource, Faculty of Agriculture, \\ Kyushu University, Fukuoka 811-2307, Japan \\ (Received June 30, 2004 and accepted July 13, 2004)
}

\begin{abstract}
In order to obtain agricultural information of Web site in different languages, the multilingual search engine system was developed for farmers of many countries. The Namazu search engine was applied to multilingual search engine method for in-site Web and Google search engine was adopted for WWW respectively. Two subsystems of the multilingual search engine system were designed to solve the language problem. One is the basic terminology translation dictionary for translation of retrieval terms, and the other is Web ASP translation service for translation of retrieval results and contents. For search of in-site Web and WWW of other country languages, firstly, farmers write a retrieval term in their mother language on the text input form of the translation dictionary, and then it is translated into the retrieval term of other languages by using the basic terminology translation dictionary. Secondly, farmers can search exactly the retrieval term in the Web site of different languages by search engine system, Namazu or Google. Finally, the retrieval results are translated into their own native languages by using the Web ASP translation service. The farmers could use much information of other country languages as well as information of native language without difficulty of language. Therefore, the amount of information available for agricultural production increased remarkably by using the multilingual search engine system.
\end{abstract}

\section{INTRODUCTION}

As a result of the development of the information technology, almost kind of information is accessible to anywhere in the world. Because of the remarkable development of Internet technology in Japan and Korea especially, farmers of each country have utilized the Internet by broadband as well as urban dwellers. They are able to obtain easily much information on the Internet and to share the agricultural information or database (Kouno and Machida, 2001; Rohrig et al., 2001; Lopes et al., 2001). We can regard WWW as the huge information database, and most of information on Web site can be used by search engine.

Search services on the Web are derived from two basic paradigms generally, directory services and query-based search engines. Directory services such as Yahoo, Einet Galaxy, Link center, and Nerd world, etc., provide a classification of WWW resources (Callery and Tracy-proulx, 1997). In contrast to directory services, query-based search

\footnotetext{
${ }^{1}$ Laboratory of Agricultural Ecology, Division of Agricultural Ecology, Department of Plant Resource, Graduate School of Bioresource and Bioenvironmental Science, Kyushu University

* Corresponding author (E-mail: knkjfam@mbox.nc.kyushu-u.ac.jp)
} 
engines such as Excite, Lycos, Inforseek, Alta Vista, Goo, and Google provide broad coverage of the Web through intensive automation of indexing and retrieval processes. These services construct databases built from robotic collection of Web pages (Jenkins et al., 1998). Because of the utilization for only information of the particular language in the existing search engine, farmers of many countries have a difficulty to get information of other country languages on the Internet. The multilingual search engine system is necessary to get agricultural information in the Web site of different language for farmers.

The current study deals with the conceptual model of multilingual search engine system. The multilingual search engine system was designed to get a lot of information of Web site in other country languages as well as native language.

\section{MATERIALS AND METHODS}

\section{The multilingual collaboration system of agricultural production}

Farmers of many countries can share crops condition, the environmental conditions in the greenhouse, running state of the equipment, cultivation log, observation log, cultivation multimedia database and agricultural information through the Internet on the multilingual collaboration system of agricultural production (Lee and Nakaji, 2003). Farmers are able to collaborate on agricultural production on the multilingual collaboration system by their own native languages.

Figure 1 shows the composition of the collaboration system with Client-Server type. The IP camera was set in the greenhouse of Kyushu University Farm, where melons were cultivated by nutrient solution culture. The crops are observed in real time on the screen of multilingual collaboration system of agricultural production by IP camera images.

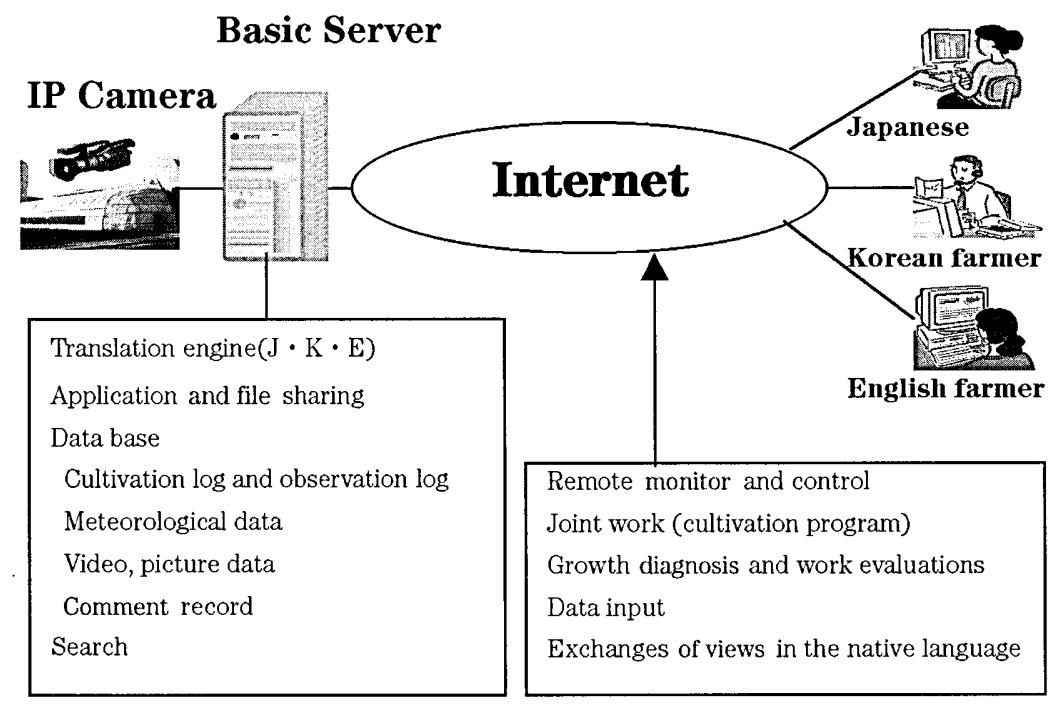

Fig. 1. The configuration of agricultural production collaboration system with Client-Server type. 
Farmers can confirm the cultivating situation and environment for agricultural facilities of specific region on the multilingual collaboration system through the Internet. The language translation system was composed of two subsystems, the basic terminology translation dictionary and the Web ASP translation service. The farmers can exchange the views and comments for crops condition in the greenhouse and appropriate work on the multilingual collaboration system by their own native languages.

The multilingual search function was used to get voluminous information of Web site in other country languages on the multilingual collaboration system of agricultural production. Figure 2 shows the multilingual search function on the screen of multilingual collaboration system. Farmers are able to utilize the multilingual search engine system on the multilingual collaboration system of agricultural production through the Internet. The search engines of two types, Namazu and Google, were used for the multilingual search engine system. Namazu is used to retrieve the information for in-site Web, and Google is used to retrieve the information for WWW.

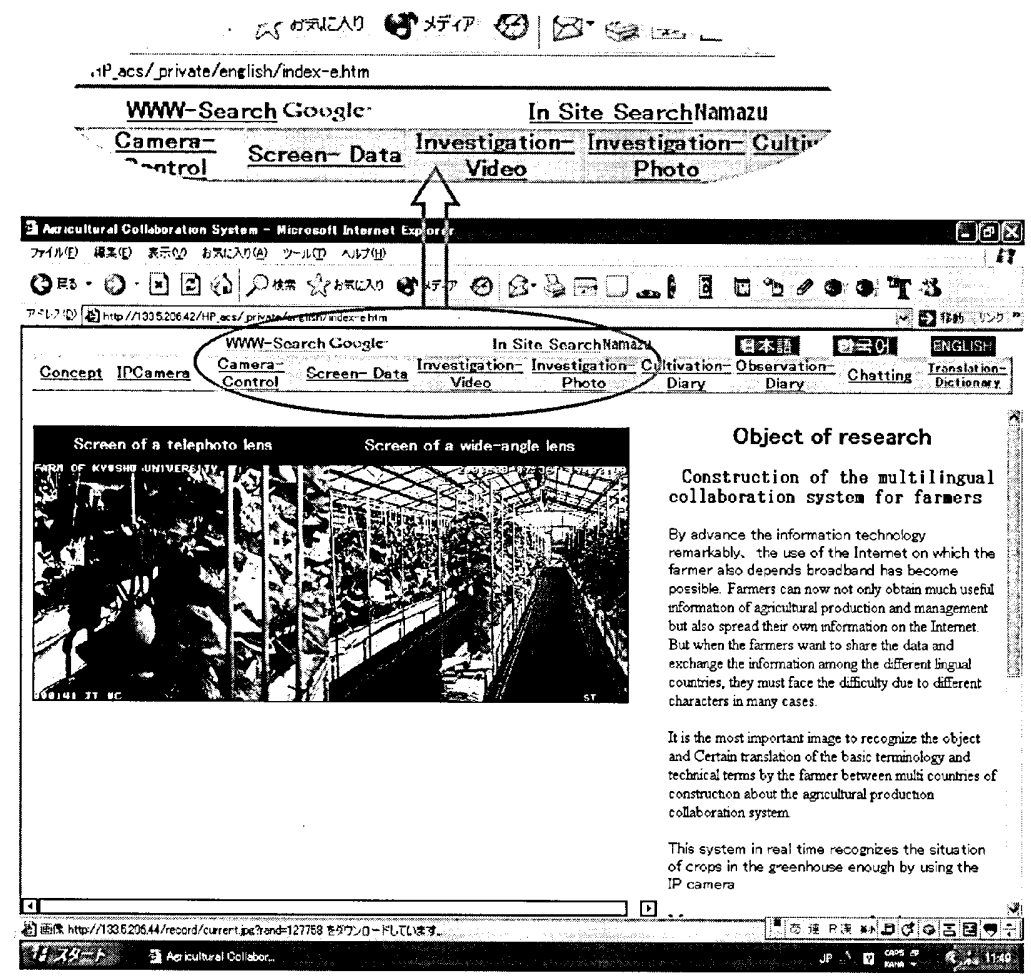

Fig. 2. Search engine function on the screen of multilingual collaboration system of agricultural production for farmers of each country.

\section{Namazu search engine system}

Namazu search engine is used for the Japanese fulltext search system intended for easy utilization. It is free software which can be downloaded from Namazu home page on 
the Internet (Namazu, 2003). It can construct the WWW fulltext search system of a small or medium scale by operating as CGI (Common Gateway Interface), and it can be also used for a personal database with network. It consists of the 'mknmz' command to make the index files and the 'namazu' command to retrieve the index files (Baba, 2003).

Figure 3 shows the flow chart of Namazu command in Namazu search engine. The Namazu search engine system has two functions "indexing" and "searching". The former makes index of text files by mknmz command described with Perl language, and the latter searches index files by namazu command described with $\mathrm{C}$ language. Namazu search system is able to retrieve the information of Web site as well as database of particular personal computer through the network.

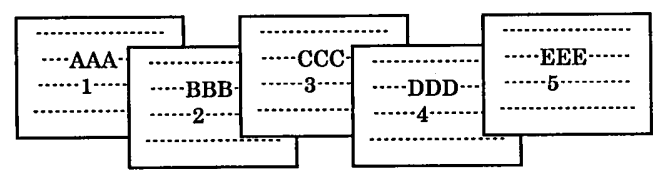

(1) To prepare the many text files

mknmz

command
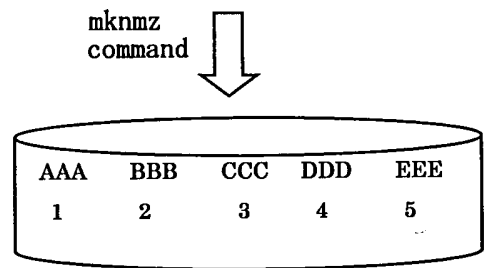

(2) To make the index

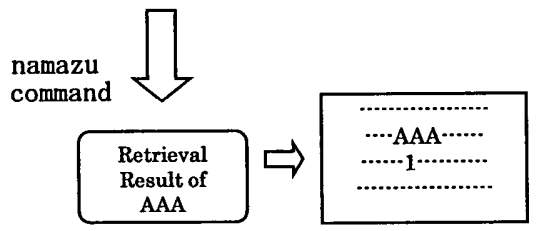

(3) To display the retrieval result

Fig. 3. The flow chart of the Namazu command.

\section{Method of multilingual search engine system}

Search function is a tool to retrieve a necessary data in enormous information easily and quickly. The query-based search engine was applied to the multilingual search engine method. To solve the language problem, the multilingual search method was carried out by two subsystems, i.e. the basic terminology translation dictionary for translation of retrieval term, and the Web ASP translation service for translation of retrieval results and contents.

In order to search the Web site in other country languages, firstly, farmers write a retrieval term in their mother language on the text input form of the basic terminology translation dictionary, and then it is translated into the retrieval term of other languages 
(Lee and Nakaji, 2004). Secondly, the translated retrieval term is inserted in the search engine of Namazu or Google. Thirdly, the retrieval results are translated in native language by using the Web ASP translation service. Finally, the farmers can choose the necessary information data of retrieval results, and obtain the retrieval contents translated into their native language by using the Web ASP translation service. As a result, farmers can get much information in the Web site of other country languages without difficulty of language by the multilingual search engine system.

\section{RESULTS AND DISCUSSION}

\section{Example of multilingual search engine method}

Figure 4 shows the flow chart to retrieve Japanese language Web site in Korean language. First of all, a Korean retrieval term is translated into the Japanese retrieval term by using the basic terminology translation dictionary. The translated Japanese retrieval term is input to the text form and retrieved by Namazu / Google search engine function. After the result of retrieval in Japanese is translated into Korean language by using the Web ASP translation service, the Korean farmer can obtain information of retrieval contents in Korean language. The Japanese farmer can retrieve the Korean Web site by the similar procedures for Google search. Farmers are able to get much agricultural information of Web site of other country languages as well as Web site of their native languages.

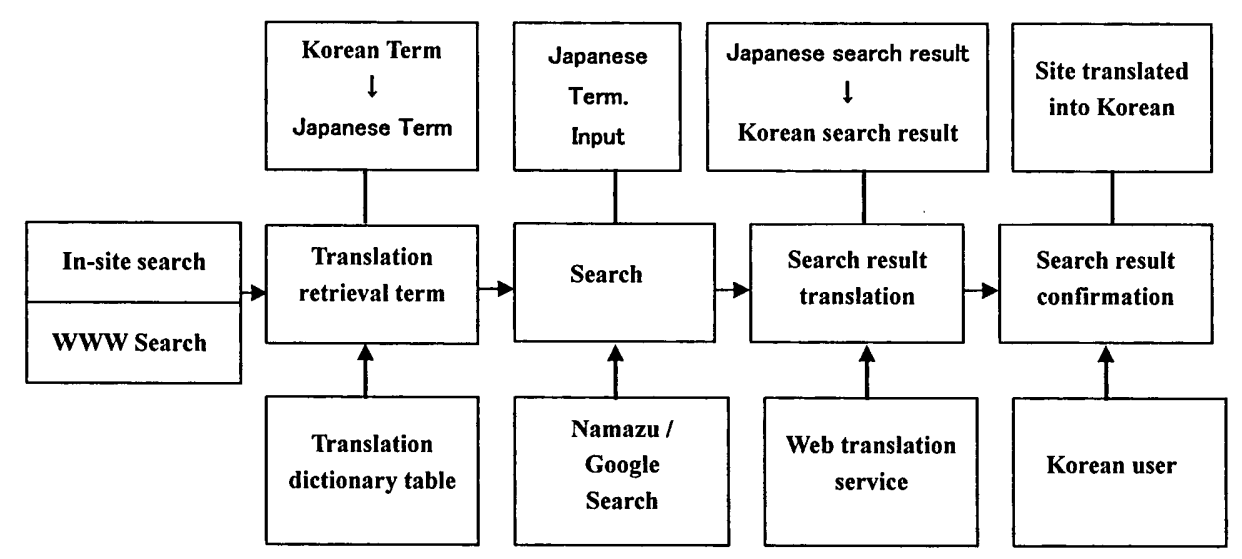

Fig. 4. The flow chart to retrieve Japanese language Web site in Korean language.

\section{Search of the in-site by Namazu}

Namazu search engine was used to search the Web of in-site of other country languages on the multilingual collaboration system. In this study, the Web of in-site means actually the site of the multilingual collaboration system of agricultural production described originally by Japanese language. Therefore, when this Web site is searched, the farmers of Korea have the problem of language. To search the information of Japanese 


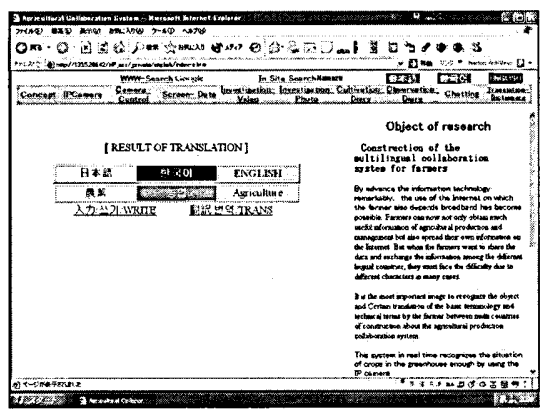

(a)

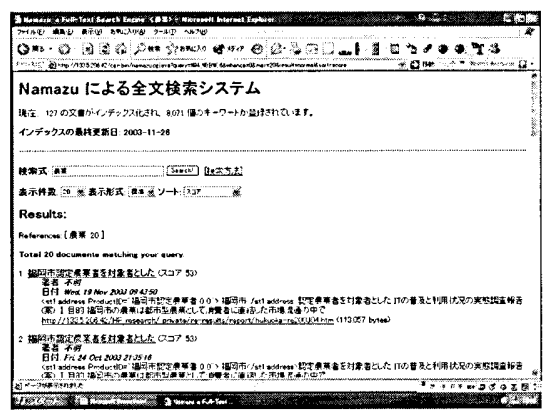

(c)

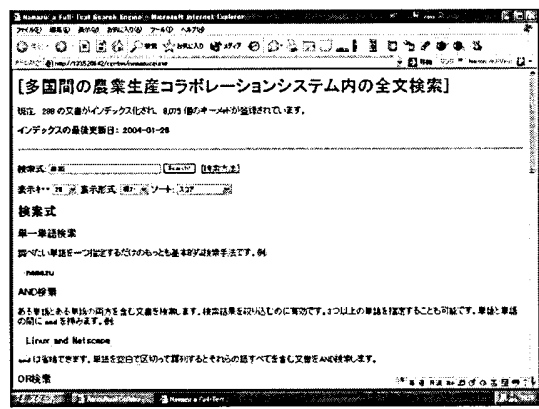

(b)

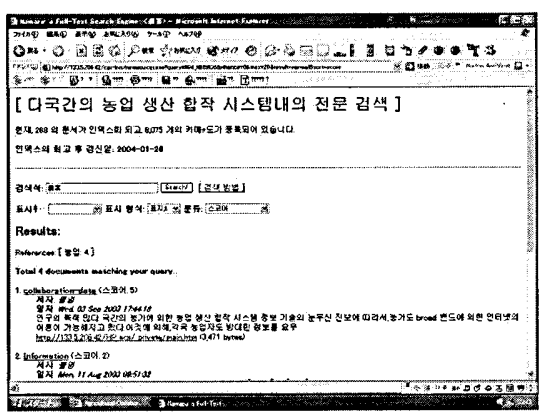

(d)

Fig. 5. Screens of the in site search by Namazu.
(a) Japanese translation of retrieval term
(b) Namazu search engine system
(c) retrieval results by Namazu
(d) Korean translation of retrieval results

language, multilingual search engine system is used for Korean farmers. Korean Farmers are able to search information of Web site of different languages by Korean language.

Figure 5 shows the search process of Namazu search engine by Korean language. The Korean farmer can search the Web of in-site of Japanese by using the basic terminology translation dictionary and the Web ASP translation service. The process is as follows: (a) The retrieval term of Korean language is translated into the retrieval term of Japanese language by using the basic terminology translation dictionary. (b) The Namazu search engine is used for Japanese fulltext search. The retrieval term translated into Japanese is inserted in the text form. (c) The retrieval results of Japanese are displayed on the screen by Namazu. (d) The retrieval results are translated from Japanese into Korean by the Web ASP translation service Mobico \& Sysmeta co.. The Korean farmer chooses the necessary information data and can get the necessary information in Korean language.

\section{Search of WWW by Google}

The Korean farmers are able to search for information on WWW of Japanese by their native languages. Figure 6 shows the search process on WWW of Japanese language by 


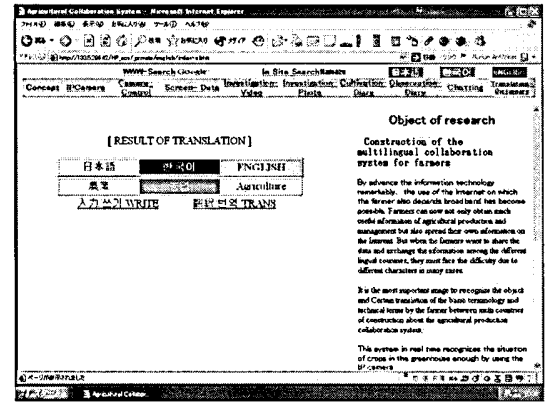

(a)

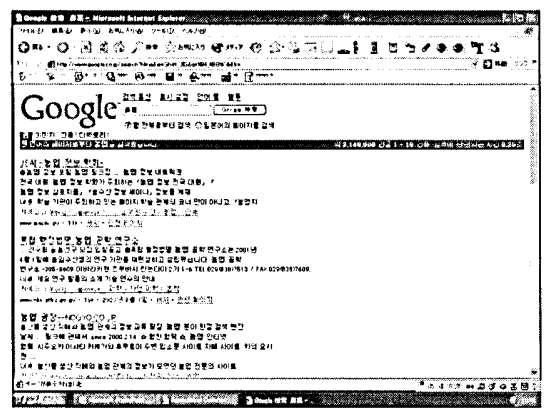

(c)

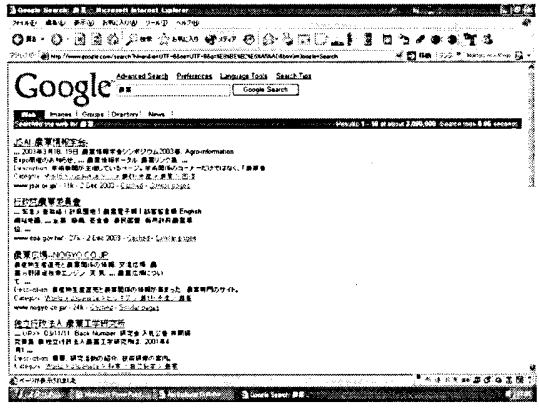

(b)

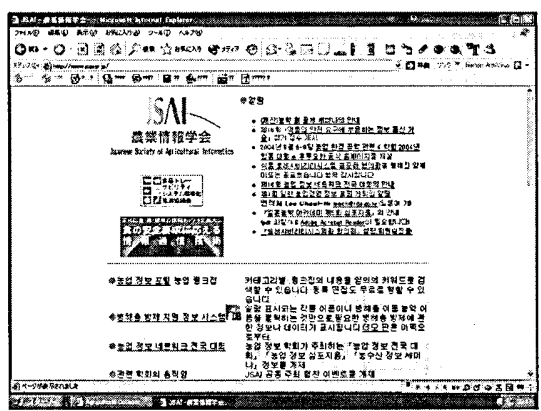

(d)

Fig. 6. Screens of the search in WWW search by Google.

(a) Japanese translation of retrieval term

(c) Korean translation of retrieval results

(b) retrieval results by Google

(d) Korean translation of retrieval contents

the Korean farmer. The process is as follows: (a) The retrieval term of Korean language is translated into the retrieval term of Japanese language by using the basic terminology translation dictionary. (b) The Google search engine is used for search of Japanese WWW. The retrieval results in Japanese language are shown as in the figure. (c) The retrieval results are translated from Japanese into Korean by the Web ASP translation service of Mobico \& Sysmeta co.. (d) The retrieval contents are translated from Japanese into Korean by the Web ASP translation service too. Japanese farmer can search the information of Web site of Korean language in the same method.

Consequently, the amount of information available for collaboration increased remarkably by using the multilingual search system.

\section{CONCLUSION}

The multilingual search system was developed to share the agricultural information in Web site of other country languages for farmers. The multilingual search engine was used not only to obain much information of Web site in other language but also to carry out the fulltext search for other language database. It is very useful to get much information of 
Web site and database in other country languages, even if the procedure of translation is slightly troublesome. Farmers could get a lot of information of the other languages as well as information of the native language without a language barrier.

\section{REFERENCES}

Baba, H. 2003 Construction and application of Namazu system, SoftBank publishing, Tokyo (in Japanese)

Callery, A. and D. Tracy-Proulx 1997 Yahoo! Cataloguing the Web. Journal of Internet Cataloging, 1(1): $57-64$

Jenkins, C., M. Jackson, P. Burden and J. Wallis 1998 Searching the World Wide Web: an evaluation of available tools and methodologies. Information and Software Technology, 39: 985-994

Kouno, T. and T. Machida 2001 I-mode cellular phone access type crop protection support system "PADB". EFITA 2001, 1: 31-36

Lee, K. O. and K. Nakaji 2003 Development of the multilingual collaboration system for agricultural production by using IP camera. In "Proceedings of the international workshop on agricultural engineering and agro-products processing toward mechanization and modernization in agriculture and rural areas, Hochiminh, Vietnam", Agricultural publishing house 2004, pp114-124

Lee, K. O. and K. Nakaji 2004 Development of the multilingual collaboration system for farmers of several countries (Part 1) - Applicaion of Basic terminology translation dictionary -. Journal of the Faculty of Agriculture, Kyushu University, 49(2): 433-440

Lopes, A. A., C. E. Cugnasca and A. M. Saraiva 2001 Remote interface for an environment control system using the internet. EFITA 2001, 2: 525-530

Rohrig, M., J. G. Hansen and P. Lassen 2001 Web-Blight implementation of a Web-based international information and decision support system for potato late blight. EFITA 2001, 1: 65-70

Google search engine 2003 http://www.google.com

Namazu search engine 2003 http://www.namazu.org/

Mobico \& Sysmeta translation-service 2003 http://www.mobico.com/ 